\title{
An Efficient Three Component Synthesis of $1 H$-Indazolo[2, 1-b]phthalazine-triones Catalyzed by Orthophosphoric Acid in Water-Phenol System
}

\author{
MANURI BRAHMAYYA ${ }^{1,2^{*}}$, REDDYMASU SREENIVASULU ${ }^{3}$, CH.CHINA \\ SATYANARAYANA ${ }^{4}$, BATTULA VENKATESWARA RAO ${ }^{1, *}$, K. N. SANTHI ${ }^{5}$, \\ M. V. RAGHUPATHIRAJU ${ }^{6}$ and M. VIJAYA ${ }^{1}$
}

${ }^{1}$ Department of Engineering Chemistry, Andhra University, Visakhapatnam-530 003, A.P., India

${ }^{2}$ Department Environamental Engineering Safety and Health, Hungkuang University, Taichung, Taiwan

${ }^{3}$ Department of Chemistry, Acharya Nagarjuna University, Guntur, India

${ }^{4}$ S K B R College, Department of Chemistry, Amalapuram, East Godavari Dist, A.P, India

${ }^{5}$ Chaitanya women's College, Department of Zoology, Gajuwaka, Viskhapatnam, India

${ }^{6}$ Department of Chemistry, D.N.R.College, Bhimavaram, West Godavari, A.P, India

manurichinna_07@yahoo.co.in

Received 9 November 2016 / Accepted 8 December 2016

\begin{abstract}
Simple method for the synthesis of $1 H$-Indazolo [2,1-b]phthalazine-triones via one pot three component condensation of phthalhydrazide, aldehyde and dimedone catalyzed by orthophosphoric acid in water-phenol system is reported. This synthetic procedure proves with extremely lower reaction timings, high yields, recyclable of catalyst and green solvent system.
\end{abstract}

Keywords: $1 H$-Indazolo[2,1- $b]$ phthalazinetrione, Multi component reactions, Orthophosphoric acid, Water-phenol system

\section{Introduction}

Multi-component reactions are attractive and interesting transformations in organic synthesis due to atom-economy, convergent character, operational simplicity, structural diversity and complexicity $^{1-7}$ these reactions are emerging as a dominating tool for the synthesis of biologically significant compounds ${ }^{8,9}$.

In recent years, the synthesis of new heterocyclic compounds has been a subject of unlimited interest due to their vast applicability. Amongst, nitrogen heterocyclic containing a phthalazine moiety was reported to possess anticonvulsant ${ }^{10}$, cardiotonic $^{11}$, vasorelaxant ${ }^{12}$ antimicrobial $^{13}$, anti-inflammatory ${ }^{14}$, antifungal ${ }^{15}$ and anticancer ${ }^{16,17}$ activities. Newly, three- 
component reactions of dimedone (5,5-dimethylcyclohexane-1,3-dione), an aldehyde and phthalhydrazide to give $1 H$-indazolo[2,1-b]phthalazine-triones have fascinated the interest of the synthetic organic chemists.

Many methods had been reported with different catalytic systems includingp-TSA ${ }^{18}$, $\mathrm{Me}_{3} \mathrm{SiCl}^{19}$, silica sulfuric acid ${ }^{20},{ }^{21} \mathrm{H}_{2} \mathrm{SO}_{4}$,cyanuric chloride ${ }^{22}$, heteropolyacids ${ }^{23}, N$-halo sulphonamides ${ }^{24}$, silica supported poly phosphoric $\operatorname{acid}^{25},{ }^{26} \mathrm{Mg}\left(\mathrm{HSO}_{4}\right)_{2}$, phosphor sulfonic $\operatorname{acids}^{27}$, nano silica sulphuric acid ${ }^{28}$, sulfonated polyethylene glycol ${ }^{29}$ and boron sulfonic $\operatorname{acid}^{30}$.

However, some of these methodologies have some experimental problems and drawbacks due to longer reaction times, requires high stoichiometric expensive reagents, hazardous solvents and struggles with a lot of workup procedures. To overcome these drawbacks, we developed an efficient and new eco-friendly methodology with better reagent orthophosphoric acid in water-phenol system for one pot three-component synthesis of $1 H$-indazolo[2,1-b]phthalazine-triones (Scheme 1).

\section{Experimental}

A mixture of phthalhydrazide (1.1 mmol), aromatic aldehyde (1.2 mmol), 5,5-dimethyl 1,3cyclohexane dimedone $(1.0 \mathrm{mmol})$, orthophosphoric acid $(0.2 \mathrm{mmol})$ and $15 \mathrm{~mL}$ of waterphenol [1:1 (in volume)] was agitated magnetically under reflux at $70{ }^{\circ} \mathrm{C}$ for a certain time (Table 1). The reaction was monitored by TLC using ethyl acetate methylene dichloride $(9: 1)$. After completion of the reaction the precipitate of reaction mixture was collected and filtered. Thereafter, it was washed with water and dried. Finally, the crude product was recrystallized from hot ethanol to yield pure products.

\section{Spectral data}

2,3,4,13-Tetrahydro-3,3-dimethyl-13-(3-methylphenyl)-1H-indazolo[1,2-b]phthalazine1,6,11-trione $\left(4 a, \mathrm{C}_{24} \mathrm{H}_{22} \mathrm{~N}_{2} \mathrm{O}_{3}\right)$

Yellow powder, m.p.: $229-230{ }^{\circ} \mathrm{C}$; IR (KBr): 1639,1616; 1419, 1356, 1,120, 993, $864 \mathrm{~cm}^{-1}$; ${ }^{1} \mathrm{H}$ NMR (400 MHz, $\left.\mathrm{CDCl}_{3}\right): 0.95\left(\mathrm{~s}, 6 \mathrm{H}, 2 \mathrm{XCH}_{3}\right), 1.86$ (s, 2H, $\mathrm{CH}_{2}$ near double bond), 2.36 $\left(\mathrm{m}, 5 \mathrm{H}, \mathrm{CH}_{2}\right.$ near keto group and was for $\left.\mathrm{Ar}-\mathrm{CH}_{3}\right), 5.1(\mathrm{~s}, 1 \mathrm{H},-\mathrm{NCH}-\mathrm{Ar}), 7.21-7.60(\mathrm{~m}, 8 \mathrm{H}$, Ar-H). ${ }^{13} \mathrm{C}$ NMR (125 MHz, $\left.\mathrm{CDCl} 3\right): \mathrm{d}=21.5,28.5,28.6,34.9,38.1,51.0,65.0,118.8$, 124.1, 127.7, 27.9, 128.0, 128.6, 129.0,129.2, 129.6, 133.4, 134.4, 136.3, 138.3, 150.7, 154.2,156.1, $192.1 \mathrm{ppm}$; MS (EI): $m / z=387.50(\mathrm{M}+\mathrm{H})^{+}$.

\section{3-(3,4-Dimethylphenyl)-2,3,4,13-tetrahydro-3,3-dimethyl-1H-indazolo[1,2- b]phthalazine-1,6,11-trione (4b, $\left.\mathrm{C}_{25} \mathrm{H}_{24} \mathrm{~N}_{2} \mathrm{O}_{3}\right)$}

Yellow powder, m.p.: $251-250{ }^{\circ} \mathrm{C}$; IR (KBr): 1,635, 1,616, 1,419, 1,357, 1,315, 1,263, $1,120,1,066,993,952,864 \mathrm{~cm}^{-1} ; 1.00\left(\mathrm{~s}, 6 \mathrm{H}, 2 \times \mathrm{CH}_{3}\right), 1.89\left(\mathrm{~s}, 2 \mathrm{H}, \mathrm{CH}_{2}\right.$ near double bond),

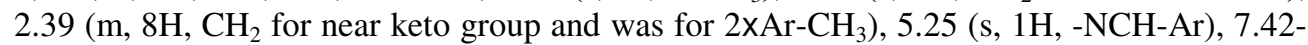
$7.62(\mathrm{~m}, 7 \mathrm{H}, \mathrm{Ar}-\mathrm{H}) .{ }^{13} \mathrm{C}$ NMR $(125 \mathrm{MHz}, \mathrm{CDCl} 3): \mathrm{d}=19.6,19.9,28.5,28.7,34.7,38.1$, 51.0, 64.9, 118.9, 124.5, 127.8, 127.9, 128.5, 129.0,129.2, 130.0, 133.4, 133.7, 134.4, 136.9, 137.2, 150.7,154.2, 156.1, $192.1 \mathrm{ppm}$; MS (EI): $\mathrm{m} / z=401.16(\mathrm{M}+\mathrm{H})^{+}$.

\section{2,3,4,13-Tetrahydro-3,3-dimethyl-13-(3-phenoxyphenyl)-1H-indazolo[1,2-} b]phthalazine-1,6,11-trione $\left(4 c, \mathrm{C}_{29} \mathrm{H}_{24} \mathrm{~N}_{2} \mathrm{O}_{4}\right)$

Yellow powder, m.p.: $188-191{ }^{\circ} \mathrm{C}$; IR (KBr): 1,635,1,616, 1,452, 1,365, 1,311, 1,272, 1,240, 1,070, 985,864 $\mathrm{cm}^{-1} ;{ }^{1} \mathrm{H}$ NMR (500 MHz, CDCl3): =1.16 (s, 3H), 1.19 (s, 3H), 2.33 (s, 2H), 
3.21 and $3.36(\mathrm{AB}$ system, $\mathrm{J}=19.0 \mathrm{~Hz}, 2 \mathrm{H}), 6.41(\mathrm{~s}, 1 \mathrm{H}), 6.88(\mathrm{dd}, \mathrm{J}=8.0,2.0 \mathrm{~Hz}, 1 \mathrm{H})$, $6.98(\mathrm{~d}, \mathrm{~J}=8.0 \mathrm{~Hz}, 2 \mathrm{H}), 7.01(\mathrm{t}, \mathrm{J}=2.0 \mathrm{~Hz}, 1 \mathrm{H}), 7.08(\mathrm{t}, \mathrm{J}=7.5 \mathrm{~Hz}, 1 \mathrm{H}), 7.22(\mathrm{~d}, \mathrm{~J}=8.0$ $\mathrm{Hz}, 1 \mathrm{H}), 7.27-7.31(\mathrm{~m}, 3 \mathrm{H}), 7.83-7.88(\mathrm{~m}, 2 \mathrm{H}), 8.27-8.30(\mathrm{~m}, 1 \mathrm{H}), 8.33-8.36(\mathrm{~m}, 1 \mathrm{H})$ ppm; ${ }^{13} \mathrm{C}$ NMR $\left(125 \mathrm{MHz}, \mathrm{CDCl}_{3}\right): \mathrm{d}=28.4,28.7,34.6,38.0,51.0,64.6,117.1,118.3$, 118.6, 119.1, 122.4, 123.4, 127.8, 128.0, 129.0, 129.1,129.7, 129.9, 133.6, 134.6, 138.4, 151.0, 154.4, 156.0,156.7, 157.6, $192.1 \mathrm{ppm} ; \mathrm{MS}(\mathrm{ESI}): \mathrm{m} / \mathrm{z}=465.11(\mathrm{M}+\mathrm{H})^{+}$.

13-(3-Bromophenyl)-2,3,4,13-tetrahydro-3,3-dimethyl-1H-indazolo[1,2b]phthalazine-1,6,11-trione $\left(4 d, \mathrm{C}_{23} \mathrm{H}_{19} \mathrm{BrN}_{2} \mathrm{O}_{3}\right)$

Yellow powder, m.p.: $223-225^{\circ} \mathrm{C}$; IR (KBr):1,635,1,618, 1,458, 1,419, 1,363, 1,309, 1,265, 1,120, 1,068, 952,864 cm ${ }_{-1}$; ${ }^{1} \mathrm{H}$ NMR (500 MHz, CDCl3): $\mathrm{d}=1.20(\mathrm{~s}, 3 \mathrm{H}), 1.21$ (s, 3H), 2.34 $(\mathrm{s}, 2 \mathrm{H}), 3.24$ and $3.41(\mathrm{AB}$ system, $\mathrm{J}=19.0 \mathrm{~Hz}, 2 \mathrm{H}), 6.39(\mathrm{~s}, 1 \mathrm{H}), 7.22(\mathrm{t}, \mathrm{J}=8.0 \mathrm{~Hz}, 1 \mathrm{H})$, $7.41(\mathrm{~d}, \mathrm{~J}=8.0 \mathrm{~Hz}, 2 \mathrm{H}), 7.47(\mathrm{t}, \mathrm{J}=2.0 \mathrm{~Hz}, 1 \mathrm{H}), 7.85-7.89(\mathrm{~m}, 2 \mathrm{H}), 8.26-8.29(\mathrm{~m}, 1 \mathrm{H})$, 8.35-8.38 (m, 1H) ppm; ${ }^{13} \mathrm{C} \mathrm{NMR}\left(125 \mathrm{MHz}, \mathrm{CDCl}_{3}\right): \mathrm{d}=28.5,28.6,34.7,38.0,50.9,64.3$, $117.1,122.8,126.4,127.8,128.1,129.0,129.8,130.2,131.9,133.7,134.6,138.7,151.2$, 154.4, 156.0,192.0 ppm; MS (ESI): $\mathrm{m} / z=452.02(\mathrm{M}+\mathrm{H})^{+}$.

2,3,4,13-Tetrahydro-3,3-dimethyl-13-[3-(trifluoro-methyl)phenyl]-1H-indazolo[1,2b]phtha lazine-1,6,11-trione ( $\left.4 e, \mathrm{C}_{24} \mathrm{H}_{19} \mathrm{~F}_{3} \mathrm{~N}_{2} \mathrm{O}_{3}\right)$

Yellow powder, m.p.: $216-217^{\circ} \mathrm{C}$; IR (KBr): 1,635,1,616, 1,359, 1,311, 1,263, 1,078, 983, $864 \mathrm{~cm}^{-1} ;{ }^{1} \mathrm{H}$ NMR(500 MHz, $\left.\mathrm{CDCl}_{3}\right): \mathrm{d}=1.21(\mathrm{~s}, 3 \mathrm{H}), 1.22(\mathrm{~s}, 3 \mathrm{H}), 2.34(\mathrm{~s}, 2 \mathrm{H}), 3.24$ and 3.43 (AB system, $\mathrm{J}=19.0 \mathrm{~Hz}, 2 \mathrm{H}), 6.49(\mathrm{~s}, 1 \mathrm{H}), 7.48(\mathrm{t}, \mathrm{J}=8.0 \mathrm{~Hz}, 1 \mathrm{H}), 7.55(\mathrm{~d}, \mathrm{~J}=8.0 \mathrm{~Hz}$, $1 \mathrm{H}), 7.60(\mathrm{~s}, 1 \mathrm{H}), 7.68(\mathrm{~d}, \mathrm{~J}=8.0 \mathrm{~Hz}, 1 \mathrm{H}), 7.85-7.89(\mathrm{~m}, 2 \mathrm{H}), 8.25-8.28(\mathrm{~m}, 1 \mathrm{H}), 8.36-8.39$ $(\mathrm{m}, 1 \mathrm{H}) \mathrm{ppm} ;{ }^{13} \mathrm{C}$ NMR $(100 \mathrm{MHz}, \mathrm{CDCl} 3): \mathrm{d}=28.3,28.8,34.7,38.1,50.9,64.3,117.1$, 123.5, $123.6(\mathrm{q}, \mathrm{J}=3.7 \mathrm{~Hz}), 125.6(\mathrm{q}, \mathrm{J}=3.6 \mathrm{~Hz}), 126.5(\mathrm{q}, \mathrm{J}=270.8 \mathrm{~Hz}), 127.8,128.2$, 128.9, 129.0, 129.2, 131.1, $131.2(\mathrm{q}, \mathrm{J}=37.5 \mathrm{~Hz}), 133.8,134.7,137.5,151.4$, 154.6, 156.1,192.1 ppm; MS (EI): $m / z=441.01(\mathrm{M}+\mathrm{H})^{+}$.

\section{3-(4-Hydroxy-3-methoxyphenyl)-3,3-dimethyl-3,4-dihydro-2H-indazolo[1,2-b]} phthalazine-1,6,11(13H)-trione (4f)

Yellow powder, m.p.: $250-253{ }^{\circ} \mathrm{C} . \mathrm{IR}\left(\mathrm{KBr}, \mathrm{cm}^{-1}\right): 3408,2958,1660,1600,1493,1359$, 1270, 1234, 1135, 1030, 790, 627; $0.98\left(\mathrm{~s}, 6 \mathrm{H}, 2 \mathrm{xCH}_{3}\right), 1.90\left(\mathrm{~s}, 2 \mathrm{H}, \mathrm{CH}_{2}\right.$ near double bond), $2.38\left(\mathrm{~s}, 8 \mathrm{H}, \mathrm{CH}_{2}\right.$ for near keto group), $3.89\left(\mathrm{~s}, 3 \mathrm{H}, \mathrm{OCH}_{3}\right), 5.21(\mathrm{~s}, 1 \mathrm{H},-\mathrm{NCH}-$ $\mathrm{Ar}), 7.40-7.65(\mathrm{~m}, 7 \mathrm{H}, \mathrm{Ar}-\mathrm{H}), 9.20(\mathrm{~s}, 1 \mathrm{H}, \mathrm{Ar}-\mathrm{OH}) ;{ }^{13} \mathrm{C} \mathrm{NMR}\left(75 \mathrm{MHz}, \mathrm{CDCl}_{3}\right): \delta=$ 28.4, 28.8, 34.6, 38.1, 51.0, 56.0, 64.8, 111.0, 114.6, 118.6, 119.2, 127.7, 128.0,128.2, $129.0,129.2,133.5,134.5,146.0,146.4,150.7,156.1,192.3 \mathrm{ppm} ; \mathrm{MS}(\mathrm{ESI}): \mathrm{m} / \mathrm{z}=$ $419.30(\mathrm{M}+\mathrm{H})^{+}$.

\section{3,3-Dimethyl-13-(3,4,5-trimethoxyphenyl)-3,4-dihydro-2H-indazolo[1,2-b]} phthalazine-1, 6, 11 (13H)-trione $\mathbf{4 g})$

Yellow powder, m.p.: 231-233 ${ }^{\circ} \mathrm{C}$. IR $\left(\mathrm{KBr}, \mathrm{cm}^{-1}\right): 2960,1656,1628,1597,1507,1466$, $1425,1361,1313,1266,1125,1000,701 ;{ }^{1} \mathrm{H} \mathrm{NMR}\left(300 \mathrm{MHz}, \mathrm{CDCl}_{3}\right): \delta=1.23(\mathrm{~s}, 3 \mathrm{H})$, $1.24(\mathrm{~s}, 3 \mathrm{H}), 2.36(\mathrm{~s}, 2 \mathrm{H}), 3.21(\mathrm{~d}, \mathrm{~J}=19.1 \mathrm{~Hz}, 1 \mathrm{H}), 3.46(\mathrm{~d}, \mathrm{~J}=19.4 \mathrm{~Hz}, 1 \mathrm{H}), 3.80(\mathrm{~s}$, $3 \mathrm{H}), 3.83(\mathrm{~s}, 6 \mathrm{H}), 6.40(\mathrm{~s}, 1 \mathrm{H}), 6.63(\mathrm{~s}, 2 \mathrm{H}), 7.83-8.37(\mathrm{~m}, 4 \mathrm{H}) \mathrm{ppm} ;{ }^{13} \mathrm{C}$ NMR $(75 \mathrm{MHz}$, $\mathrm{CDCl} 3): \delta=28.1,29.0,34.6,38.1,51.0,56.2$, 60.7, 65.0, 104.6, 118.3, 127.7, 128.0, $128.9,129.0,131.8,133.6,134.6,138.3,150.9,153.4,154.5,156.1,192.2$ ppm; MS(ESI): $m / z=462.04(\mathrm{M}+\mathrm{H})^{+}$. 
13-(3-Chlorophenyl)-3,3-dimethyl-3,4-dihydro-2H-indazolo[1,2-b]phthalazine-1, 6, 11(13H)-trione $(\mathbf{4 h})$

Yellow powder, m.p.: 206-207 ${ }^{\mathrm{O}} \mathrm{C}$. IR $\left(\mathrm{KBr}, \mathrm{cm}^{-1}\right): 3069,2957,2872,1657,1626,1578$, 1464, 1360, 1310, 1268, 1145, 788, 701, 677; ${ }^{1} \mathrm{H}$ NMR (300 MHz, $\left.\mathrm{CDCl}_{3}\right): \delta=1.21(\mathrm{~s}, 6 \mathrm{H})$, $2.34(\mathrm{~s}, 2 \mathrm{H}), 3.21(\mathrm{~d}, \mathrm{~J}=19.1 \mathrm{~Hz}, 1 \mathrm{H}), 3.41(\mathrm{~d}, \mathrm{~J}=19.1 \mathrm{~Hz}, 1 \mathrm{H}), 6.40(\mathrm{~s}, 1 \mathrm{H}), 7.24-7.86$ $(\mathrm{m}, 6 \mathrm{H}), 8.24-8.37(\mathrm{~m}, 2 \mathrm{H}) \mathrm{ppm} ;{ }^{13} \mathrm{C} \mathrm{NMR}\left(75 \mathrm{MHz}, \mathrm{CDCl}_{3}\right): \delta=28.5,28.6,34.7,38.0$, 50.9, 64.3, 117.9, 125.8,127.0, 127.7, 128.1, 128.9, 129.0, 130.0, 133.7, 134.6, 138.5, 151.2, 154.4, 156.0, $192.1 \mathrm{ppm}$; MS (ESI): $\mathrm{m} / z=407.10(\mathrm{M}+\mathrm{H})^{+}$.

3,4-Dihydro-3,3-dimethyl-13-(2-chlorophenyl)-2H-indazolo[2,1-b]phthalazine-1,6, $11(13 H)$-trione $(4 i)$

Yellow powder, m.p.: $265-267{ }^{\circ} \mathrm{C}$; IR $\left(\mathrm{KBr}, \mathrm{cm}^{-1}\right)$ : 2956, 1663, 1625; ${ }^{1} \mathrm{H}$ NMR $(300 \mathrm{MHz}$, $\left.\mathrm{CDCl}_{3}\right): 1.22(3 \mathrm{H}, \mathrm{s}, \mathrm{Me}), 1.23(3 \mathrm{H}, \mathrm{s}, \mathrm{Me}), 2.33\left(2 \mathrm{H}, \mathrm{s}, \mathrm{CH}_{2} \mathrm{CO}\right), 3.24$ and $3.42(2 \mathrm{H}, \mathrm{AB}$ system, $\left.\mathrm{J}=19.1 \mathrm{~Hz}, \mathrm{CH}_{\mathrm{a}} \mathrm{H}_{\mathrm{b}} \mathrm{CO}\right), 6.69(1 \mathrm{H}, \mathrm{s}, \mathrm{CHN}), 7.25-8.40(8 \mathrm{H}, \mathrm{m}, \mathrm{Ph}) ;{ }^{13} \mathrm{C}$ NMR $\left(75 \mathrm{MHz}, \mathrm{CDCl}_{3}\right)$ : 28.4, 28.8, 34.6, 38.0, 50.9, 64.0, 116.7, 127.2, 127.6, 128.0, 128.7, 129.0, 129.9, 130.5, 132.6, 133.0, 133.6, 134.5, 151.9, 154.2, 156.2, 192.1; MS (ESI): $m / z=407.08(\mathrm{M}+\mathrm{H})^{+}$.

\section{Results and Discussion}

Here, we report one pot, three component condensation of phthalhydrazide $\mathbf{2}$, aromatic aldehydes (1a-1i) and dimedone $\mathbf{3}$ for the synthesis of aromatic $1 H$-indazolo[2,1-b] phthalazine-triones 4a-4i using orthophosphoric acid as an important catalyst under water phenol system in good to excellent yields and short reaction times (Scheme 1).

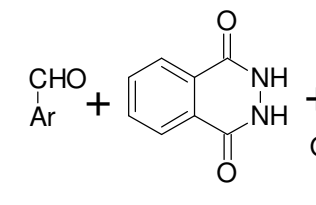

$1 \mathrm{a}-\mathrm{i}$<smiles>CC1(C)CC(=O)CC(=O)C1</smiles>

3

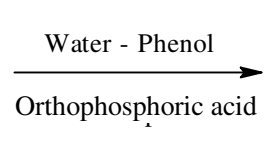

\section{Scheme 1}<smiles>CC1(C)CC(=O)C2=C(C1)n1c(=O)c3ccccc3c(=O)n1C2Br</smiles>

$4 a-i$

In order to optimize the reaction conditions, phthalhydrazide $(1.11 \mathrm{mmol})$, methoxy benzaldehyde $(1.20 \mathrm{mmol})$ and dimedone $(1.00 \mathrm{mmol})$ were used as model substrates (Table 2, entry 4a). To evaluate the catalyst effect, first of all, the reaction was performed in water system. But no product was formed. Further, to optimize the reaction conditions, the reaction was carried out in presence of acidic catalysts such as hydrochloric acid, fuming nitric acid, acetic acid and ortho phosphoric acid in different solvent systems. Whereas the reaction had failed in the presence of strong acids like $\mathrm{HCl}, \mathrm{HNO}_{3}$ as well as weak acetic acid. Now we had chosen water-phenol system (1:1) in the presence of orthophosphoric acid catalyst gave excellent yield $95 \%$ in only 15 min.time (Table 1).

Table 1. Optimization of the reaction conditions for the synthesis of aromatic $1 H$-indazolo [2,1-b] phthalazine-triones

\begin{tabular}{cccc}
\hline Entry & Catalyst, mol\% & Time, min & Yield, \% \\
\hline 1 & Water & 60 & 0 \\
2 & Water $-\mathrm{HCl}(0.21 \mathrm{mmol})$ & 60 & 0 \\
3 & Water-MeOH $(1: 1) / \mathrm{HCl}(0.2 \mathrm{mmol})$ & 60 & 15 \\
4 & Water - Phenol $(1: 1) / \mathrm{AcOH}(0.21 \mathrm{mmol})$ & 60 & 5 \\
\hline \multicolumn{2}{r}{} & & Contd $\ldots$.
\end{tabular}




\begin{tabular}{cccc}
\hline 5 & Water-Phenol $(1: 1) / \mathrm{HNO}_{3}$ & 90 & 10 \\
6 & Water-Phenol $(1: 1) / \mathrm{H}_{2} \mathrm{SO}_{4}(0.2 \mathrm{mmol})$ & 90 & 30 \\
7 & Water $/ \mathrm{H}_{3} \mathrm{PO}_{4}(0.2 \mathrm{mmol})$ & 30 & 0 \\
8 & Water-DCM $(1: 1) / \mathrm{H}_{3} \mathrm{PO}_{4}(0.2 \mathrm{mmol})$ & 30 & 0 \\
9 & Water-THF $(1: 1) / \mathrm{H}_{3} \mathrm{PO}_{4}(0.2 \mathrm{mmol})$ & 30 & 0 \\
10 & Water-DMF $(1: 1) / \mathrm{H}_{3} \mathrm{PO}_{4}(0.2 \mathrm{mmol})$ & 30 & 0 \\
11 & Water-benzene $(1: 1) / / \mathrm{H}_{3} \mathrm{PO}_{4}(0.2 \mathrm{mmol})$ & 30 & 0 \\
12 & Water-toluene $(1: 1) / \mathrm{H}_{3} \mathrm{PO}_{4}(0.2 \mathrm{mmol})$ & 30 & 0 \\
13 & Water $-\mathrm{MeOH}(1: 1) / \mathrm{H}_{3} \mathrm{PO}_{4}(0.2 \mathrm{mmol})$ & 25 & 60 \\
14 & Water-Phenol $(1: 1) / \mathrm{H}_{3} \mathrm{PO}_{4}(0.1 \mathrm{mmol})$ & 20 & 80 \\
15 & Water-Phenol $(1: 1) / \mathrm{H}_{3} \mathrm{PO}_{4}(0.2 \mathrm{mmol})$ & 15 & 95 \\
\hline
\end{tabular}

There was no particular effect of substituents on aromatic aldehydes to pull back the reaction or to stop the reaction. All the reactions were found to be completed irrespective of electron with drawing or electron donating groups present in the aromatic aldehydes and afforded corresponding products at higher yields with less reaction time. The results were summarized below (Table 2).

Table 2. Synthesis of $1 H$-indazolo-[2,1-b]phthalazinetrione derivatives using orthophosphoric acid as a catalyst (Scheme 1)

\begin{tabular}{|c|c|c|c|c|}
\hline Entry & $\mathrm{Ar}$ & Product & Time, min & Isolated Yield, \% \\
\hline $1 \mathrm{a}$ & & $4 a$ & 15 & 91 \\
\hline $1 b$ & & $4 b$ & 20 & 92 \\
\hline $1 \mathrm{c}$ & & $4 c$ & 20 & 92 \\
\hline $1 d$ & & $4 d$ & 13 & 94 \\
\hline $1 \mathrm{e}$ & & $4 \mathrm{e}$ & 12 & 87 \\
\hline 1f & & $4 \mathrm{f}$ & 10 & 93 \\
\hline
\end{tabular}




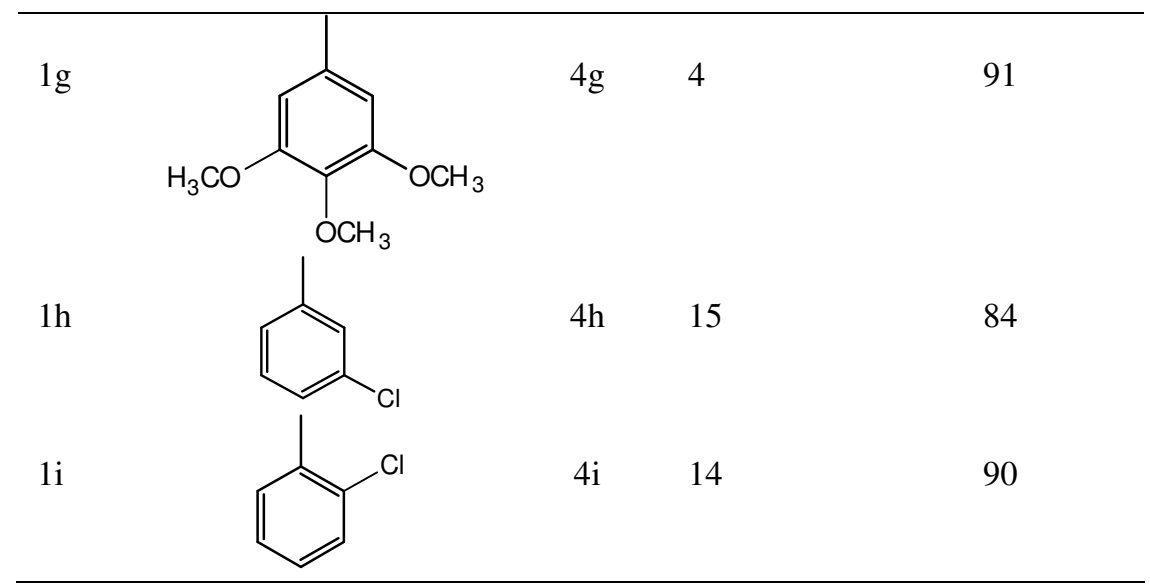

\section{Conclusion}

An efficient, one pot and three-component condensation was developed by simple, short experimental procedures to attained the $1 H$-Indazolo[2,1- $b]$ phthalazine-triones. The reaction had been catalysed by orthophosphoric acid in a semi-miscible water phenol solvent system (1:1) was environmental benign, eco friendly, inexpensive practical procedure for the synthesis of $1 H$-Indazolo[2, 1-b]phthalazine-triones compounds.

\section{Acknowledgement}

Authors are very thankful to K.V.K, DKR, IICT and Hyderabad, Central university of Hyderabad and Andhra University, Visakhapatnam-530003, Andhra Pradesh, India for the analytical support.

\section{References}

1. Zhu J and Bienayme H, Multicomponent ReactionsWein-heim (Wiley-VCH), 2005.

2. Domling A, Chem Rev., 2006, 106(1), 17-89; DOI:10.1021/cr0505728

3. Ramón D J and Yus M, Angew Chem Int Ed., 2005, 44(11), 1602-1634;

DOI:10.1002/anie.200460548

4. Simon C, Constantieux T and Rodriguez J, Eur J Org Chem., 2004, 24, 4957-4980; DOI:10.1002/ejoc.200400511

5. Orru R V A and Greef M D, Synthesis, 2003, 10, 1471-1499; DOI:10.1055/s-200340507

6. Bienayme H, Hulme C, Oddon G and Schmitt P, Chem Eur J., 2000, 6(18), 33213329; DOI:10.1002/1521-3765(20000915)6:18<3321::AID-CHEM3321>3.0.CO;2-A

7. Ulaczyk-Lesanko A and Hall D G, Curr Opin Chem Biol., 2005, 9(3), 266-276; DOI:10.1016/j.cbpa.2005.04.003

8. Weber L, Curr Med Chem., 2002, 9(23), 2085-2093; DOI:10.2174/0929867023368719

9. Hulme C and Gore V, Curr Med Chem., 2003, 10(1), 51-80; DOI:10.2174/0929867033368600

10. Grasso S, De Sarro G, Micale N, Zappala M, Puia G, Baraldi M and Demicheli C, $J$ Med Chem., 2000, 43(15), 2851-2859; DOI:10.1021/jm001002x

11. Nomoto Y, Obase H, Takai H, Teranishi M, Nakamura J and Kubo K, Chem Pharm Bull., 1990, 38(8), 2179-2183; DOI:10.1248/cpb.38.2179 
12. Watanabe N, Kabasawa Y, Takase Y, Matsukura M, Miyazaki K, Ishihara H, Kodama $\mathrm{K}$ and Adachi H, J Med Chem., 1998, 41(18), 3367-3372; DOI:10.1021/jm970815r

13. El-Sakka S S, Soliman A H, Imam A M, Afinidad, 2009, 66, 167-172.

14. Sinkkonen J, Ovcharenko V, Zelenin K N, Bezhan I P, Chakchir B A, Al-Assar F and Pihlaja K, Eur J Org Chem., 2002, 13, 2046-2053; DOI:10.1002/10990690(200207)2002:13<2046::AID-EJOC2046>3.0.CO;2-C

15. Ryu C K, Park R E, Ma MY and Nho J H, Bioorg Med Chem Lett., 2007, 17(9), 2577-2580; DOI:10.1016/j.bmcl.2007.02.003

16. Li J, ZhaoY F, Yuan XY, Xu J X and Gong P, Molecules, 2006, 11(7), 574-582; DOI:10.3390/11070574

17. Ashraf F W, Aly A A, Mohamed S B and Nora S M, Der Pharm Chemica., 2013, 5, 82-89.

18. Sayyafi M, Seyyedhamzeh M, Khavasi H R and Bazgir A, Tetrahedron, 2008, 64(10), 2375-2378; DOI:10.1016/j.tet.2008.01.006

19. Nagarapu L, Bantu R and Mereyala H B, J Heterocyclic Chem., 2009, 46, 728-731; DOI:10.1002/jhet.135

20. Shaterian H R, Ghashang M and Feyzi M, App Catal A, 2008, 345(2), 128-133; DOI:10.1016/j.apcata.2008.04.032

21. Khurana J M and Magoo D, Tetrahedron Lett., 2009, 50(52), 7300-7303; DOI:10.1016/j.tetlet.2009.10.032

22. Wang, Ma W W, Wu L Q and Yan F L, J Chin Chem Soc., 2010, 57, 1341-1343.

23. Wang H J, Zhang X N and Zhang Z H, Monatsh Chem., 2010, 141, 425-430; DOI:10.1007/s00706-010-0283-7

24. Ghorbani-Vaghei R, Karimi-Nami R, Toghraei-Semiromi Z, Amiri M and Ghavidel M, Tetrahedron., 2011, 67(10), 1930-1934; DOI:10.1016/j.tet.2011.01.024

25. Shaterian H R, Hosseinian A and Ghashang M, Arkivoc, 2009, 59-67.

26. Shaterian H R, Khorami F, Amirzadeh A, Doostmohammadi R and Ghashang M, $J$ Iran Chem Res., 2009, 2, 57-62.

27. Rezakiasat A, Mouradzadegun A, Saghanezhad S J, J Serb Chem Soc., 2013, 78, 469-473.

28. Hamidian H, Fozooni S, Hassankhani A and Mohammadi S Z, Molecules., 2011, 16(11), 9041-9048; DOI:10.3390/molecules16119041

29. Hasaninejad A, Zare A and Shekouhy M, Tetrahedron., 2011, 67(2), 390-400; DOI:10.1016/j.tet.2010.11.029

30. Soheilizad M, Adib M, Sajjadifar S, Monatsh Chem., 2014, 145, 1353-1356; DOI:10.1007/s00706-014-1194-9 